\title{
A Systematic Review and Meta-analysis of Prevalence of Biopsy-Proven Lupus Nephritis
}

\author{
Hong WANG, ${ }^{1,2}$ Yi-le REN, ${ }^{3}$ Jun CHANG, ${ }^{4}$ Luo GU, ${ }^{5}$ Ling-Yun SUN ${ }^{1}$ \\ ${ }^{1}$ Department of Rheumatology and Immunology, Drum Tower Clinical Medical College of Nanjing Medical University, Nanjing, Jiangsu, China \\ ${ }^{2}$ Department of Nephrology and Rheumatology, The Second Affiliated Hospital of Wenzhou Medical University, Wenzhou, Zhejiang, China \\ ${ }^{3}$ Department of Rheumatology and Immunology, Lian Yun-gang Clinical Medical College of Nanjing Medical University, Nanjing, Jiangsu, China \\ ${ }^{4}$ Department of Rheumatology and Immunology, The Second Affiliated Hospital of Nanjing Medical University, Nanjing, Jiangsu, China \\ ${ }^{5}$ Department of Biochemistry and Molecular Biology, Drum Tower Clinical Medical College of Nanjing Medical University, Nanjing, Jiangsu, China
}

\begin{abstract}
Objectives: This study aims to conduct a meta-analysis to clarify the epidemiologic characteristics of biopsy-proven lupus nephritis (BPLN), including those relating to its prevalence and prognosis.

Patients and methods: A literature search for relevant studies was conducted in the electronic databases of PubMed, Google Scholar, Embase, and Cochrane trial register. The following search terms were used for original articles published between January 1982 and April 2016: "lupus nephritis" or systemic lupus erythematosus ('SLE') or 'systemic lupus erythematous' and "pathology" or 'epidemiology' or prevalence or incidence. Pooled estimates with $95 \%$ confidence intervals were calculated.

Results: Nineteen studies were included (mean age of SLE patients at renal biopsy: $~ 30$ years). Of total BPLN patients, $85 \%$ were females. BPLN developed in $29 \%$ of SLE patients, and accounted for $60 \%$ of secondary glomerular diseases in renal biopsy databases. BPLN prevalence among SLE patients was higher in Saudi Arabia compared with pooled Europe/USA data ( $43 \%$ vs $26 \%, p<0.05$ ). Pooled BPLN prevalence among secondary glomerular diseases patients was higher in Asian/Latin American countries than in Europe (63\% vs $34 \%$, $\mathrm{p}<0.05$ ). Overall five-, 10 - and 20 -year survival rates of BPLN patients were $94 \%, 86 \%$, and $71 \%$, respectively, which were higher than those before $1995(84 \%, 72 \%$, and $52 \%$, respectively) and lower than those after 1995 (96\%,89\%, and 80\%, respectively) (all $p<0.05)$. Class IV nephritis, present in $40 \%$ of BPLN patients, was a risk factor for renal failure that contributed to poor prognosis.

Conclusion: Lupus nephritis is a common complication of young female patients with SLE, and the most prevalent etiology of secondary glomerular diseases. Attention should be paid to class IV nephritis due to its high frequency and association with poor prognosis.

Keywords: Biopsy-proven lupus nephritis; epidemiology; meta-analysis.
\end{abstract}

Systemic lupus erythematosus (SLE) is a chronic autoimmune disorder that predominantly affects younger females. ${ }^{1-3}$ The disorder can involve many organ systems of the body, and manifests clinically in a relapsing and remitting manner. ${ }^{1-3}$ SLE is characterized by the production of autoantibodies directed against nuclear and cytoplasmic antigens, and although its etiology is not wellunderstood, numerous genetic, epigenetic, and environmental risk factors have been identified. ${ }^{1-3}$ Clinical management is based on disease severity, and includes the use of anti-inflammatory drugs (steroidal and nonsteroidal), immunosuppressive drugs, and biologic agents.

Lupus nephritis (LN) is a severe complication of SLE that has a broad spectrum of clinical and pathologic features, leading to various prognoses among patients with SLE. ${ }^{4}$ Silent nephritis, now recognized as a serious pathologic abnormality of the kidney, presents in some patients with SLE, in the absence of abnormal urinalysis findings

Received: June 30, 2016 Accepted: November 24, 2016 Published online: July 25, 2017

Correspondence: Luo Gu, MD. Department of Biochemistry and Molecular Biology, Drum Tower Clinical Medical College of Nanjing Medical University, 210008 Nanjing, Jiangsu, China. Tel: +86-25-86862016 e-mail: drguluo@hotmail.com

○2018 Turkish League Against Rheumatism. All rights reserved. 
or other clinical features such as elevated serum creatinine and hypertension. ${ }^{5}$ Biopsy of the kidney is thus considered to be of prime importance in some patients with SLE, as accurate diagnosis of biopsy-proven lupus nephritis (BPLN) allows for the instigation of an appropriate treatment strategy that can improve the survival rates of patients with SLE. 6,7

However, the prognosis of LN still varies greatly from region to region, and is likely to be affected by genetic, racial, and other epidemiologic factors. ${ }^{4,8}$ The identification of epidemiologic factors associated with BPLN will improve our understanding of this disorder, and may facilitate the diagnosis and management of this complication of SLE. To the best of our knowledge, there are only limited data relating to the epidemiologic characteristics of BPLN. Therefore, in this study, we aimed to conduct a meta-analysis to clarify the epidemiologic characteristics of BPLN, including those relating to its prevalence and prognosis.

\section{PATIENTS AND METHODS}

This study was conducted at Nanjing Medical University, China between December 2014 to April 2016. Electronic searches were performed using the PubMed, Google Scholar, Embase, and Cochrane trial register databases with the keywords "lupus nephritis" and "pathology", without restriction on settings or language. Original articles were identified which were published between January 1982 and April 2016. In addition, manual searches of reference lists were also conducted to minimize the chances of missing any articles. The following articles were excluded: review articles; case reports; repeat publications and duplications; non human studies; pediatric studies; basic science studies pertaining only to the mechanism of LN; studies relating only to repeated renal biopsy; articles focusing only on one type of LN or proliferative nephritis; articles in which renal biopsy was not routinely performed on most patients with $\mathrm{LN}$; articles focusing on all kidney diseases without discriminating between primary glomerulonephritis and secondary glomerulonephritis; and studies relating to prognosis that did not provide specific values for survival rates (Figure 1).
Two investigators independently reviewed the studies to assess their eligibility for inclusion, and independently extracted data from the selected studies. Disagreements were resolved by consensus. To describe and analyze the epidemiologic characteristics, the following data were extracted: geographic area; patient age; proportion of female patients; numbers of patients with BPLN, LN, SLE or secondary glomerular diseases (SGD); pathologic profiles of BPLN; survival rates of patients with BPLN at five, 10 and 20 years; risk factors for renal failure in patients with BPLN; and causes of death in patients with BPLN.

\section{Statistical analysis}

Statistical analyses were performed using STATA 12.0 meta-analysis software (StataCorp, College Station, TX, USA). Based on the crude numbers extracted from the individual studies, pooled estimates with 95\% confidence intervals (CIs) were established of the following epidemiologic data: prevalence rate of BPLN among patients with SLE or SGD, proportion of female patients with BPLN, proportion of patients with BPLN and class IV nephritis, and survival rates of patients with BPLN at 5,10 and 20 years. Fixed effect model was used if heterogeneity $\left(I^{2}\right)$ was less than 50\%; otherwise, random effect model was applied. $\mathrm{P}$ values $<0.05$ were taken to be statistically significant. To estimate the influence of individual studies on the summary effect estimate (i.e. sensitivity analysis), metaanalysis estimates were re-computed omitting one study at a time.

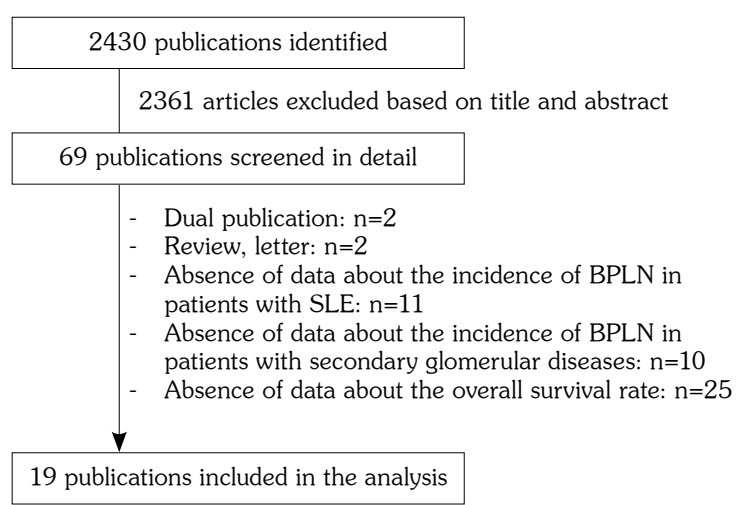

Figure 1. Flow diagram illustrating the literature search strategy. BPLN: Biopsy-proven lupus nephritis; SLE: Systemic lupus erythematosus. 


\section{RESULTS}

Of 2,430 articles initially identified by the literature search, 2,361 were excluded on screening of the title and abstract. Detailed analysis of the full text of the remaining 69 articles identified 19 that met the criteria for inclusion in our analysis (Figure 1). These 19 articles were published between 1994 and 2012, and included patients from 14 different countries. The included studies covered three study fields: five articles ${ }^{9-13}$ described the prevalence rate of BPLN among patients with SLE (Table 1); nine articles ${ }^{14-22}$ described the prevalence rate of BPLN among patients with SGD that were included in a renal biopsy database (Table 2); and six articles ${ }^{13,23-27}$ examined the prognosis of patients with BPLN, and investigated the factors associated with prognosis (Table 3 ). Two articles ${ }^{15,19}$ were serial studies carried out from January 1990 to January 2002 and from January 2003 to October 2006, respectively, and were based on the same renal biopsy database in Bahrain; therefore, the data in these two studies were combined for the purposes of this analysis. One study ${ }^{13}$ investigated both the prevalence rate of BPLN among patients with SLE and the prognosis of patients with BPLN.

The prevalence rate of BPLN among patients with SLE ranged from 16.9 to $42.8 \%$ in five studies, ${ }^{9-13}$ corresponding to a total of 815 cases of BPLN among 2,781 patients with SLE (Table 1). The overall proportion was 29\% (95\% CI: $20-38 \%$, $I^{2}=96.9 \%, \mathrm{p}<0.001$ ) (Figure 2a). In the same five studies, ${ }^{9-13}$ the prevalence rate of $\mathrm{LN}$ among patients with SLE ranged from 21.2 to $47.9 \%$, and the pooled rate was 34\% (95\% CI: $25-44 \%$, $\left.I^{2}=96.6 \%, \mathrm{p}<0.001\right)$.

These five studies $^{9-13}$ included patients from five different countries: three were based in Europe (France, Hungry, and Spain), one in the USA, and one in Saudi Arabia. The pooled rate of BPLN among patients with SLE in Europe and the USA was $26 \%$ (95\% CI: $18-33 \%, I^{2}=94.7 \%$, $\mathrm{p}<0.001$ ); this was significantly lower than the rate of 43\% (95\% CI: 39-47\%) in Saudi Arabia, but not significantly different from the pooled rate of all five countries, based on comparisons of $95 \%$ CIs (Figure 2a). Similarly, the pooled prevalence rate of LN among patients with SLE in Europe and the USA was $31 \%\left(95 \% \mathrm{CI}: 23-38 \%, I^{2}=93.8 \%\right.$, $\mathrm{p}<0.001$ ), significantly lower than the single rate

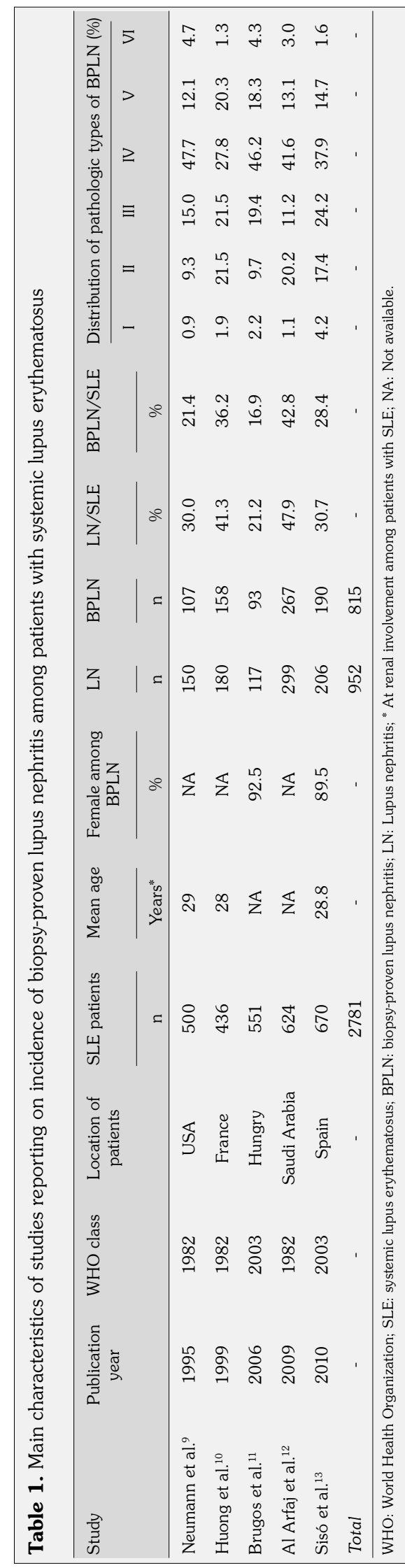


Arch Rheumatol
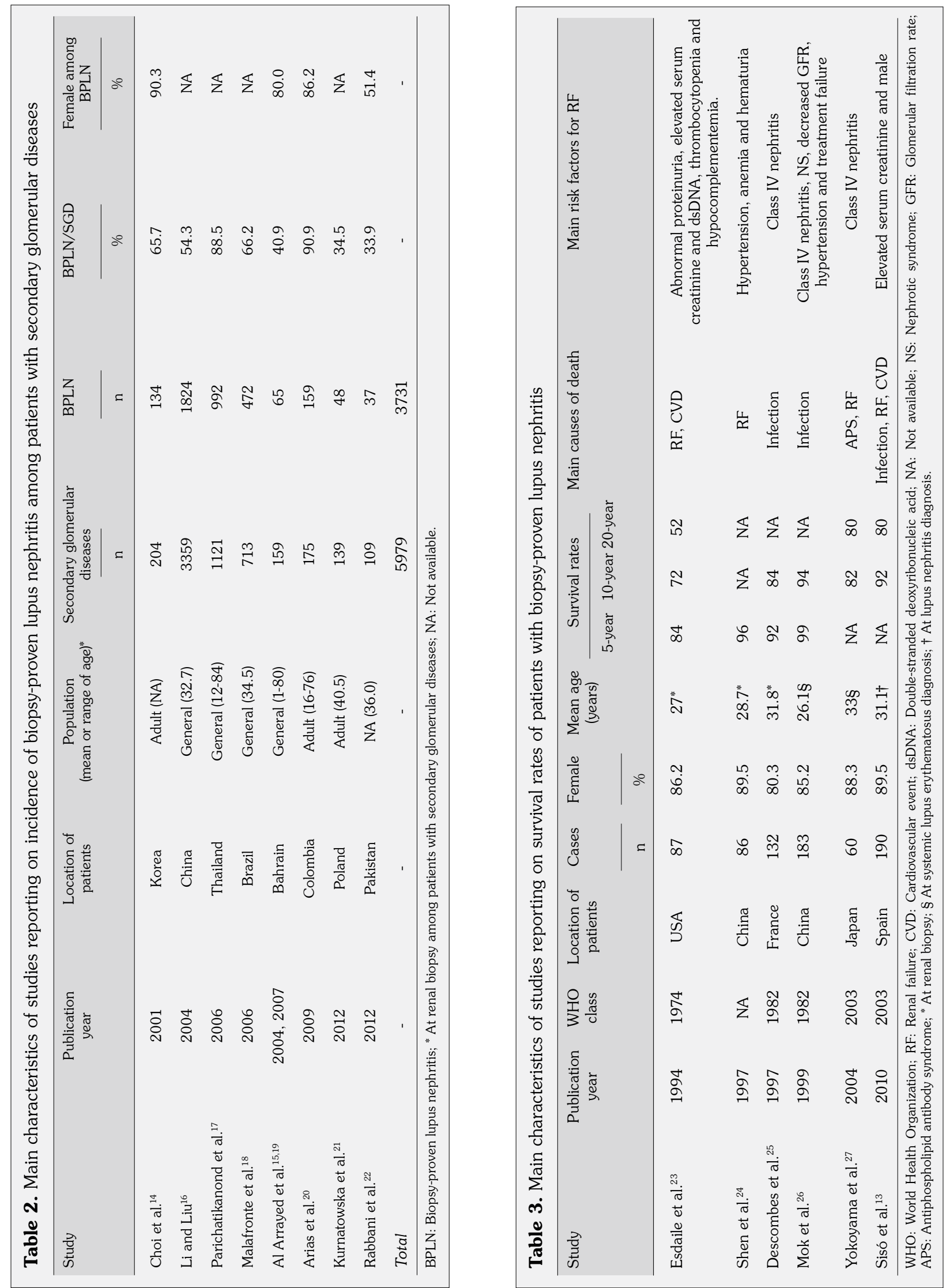
of 48\% (95\% CI: 44-52\%) in Saudi Arabia, but not significantly different from the pooled rate of all five countries, based on comparisons of $95 \%$ CIs.

Eight studies ${ }^{14-22}$ reported the prevalence rate of BPLN among patients with SGD, corresponding to a total of 3,731 patients with LN among 5,979 patients with SGD in eight renal biopsy databases (Table 2). The pooled rate of BPLN among patients with SGD was 60\% (95\% CI: $45-74 \%, I^{2}=99.3 \%, p<0.001$ ) (Figure 2b). Although the prevalence rate of BPLN among patients with SGD varied greatly from 33.9 to $90.9 \%$ in these eight studies, BPLN was consistently the most common underlying cause of SGD in each study, occurring more frequently than other potential causes such as diabetic nephropathy and Henoch-Schönlein purpura.

These eight studies ${ }^{14-22}$ were of patients from eight different countries: seven were conducted in Asia and Latin America, and one in Europe (Poland). The pooled rate of BPLN among patients with SGD in Asia and Latin America was 63\% (95\% CI: 48-79\%, $I^{2}=99.4 \%, \mathrm{p}<0.001$ ); this was significantly higher than the single rate of $34 \%$ (95\% CI: 27-42\%) in Poland, but not significantly different from the pooled rate of all eight countries, based on comparisons of 95\% CIs (Figure 2b).

(a)

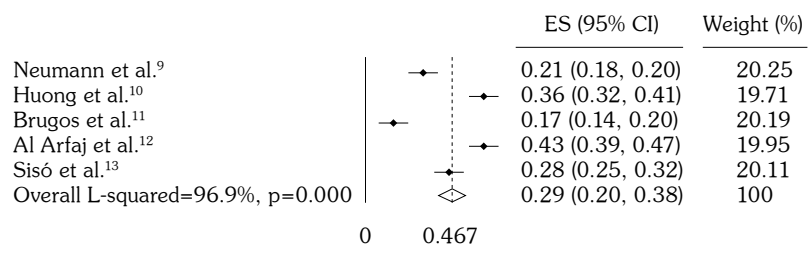

(c)

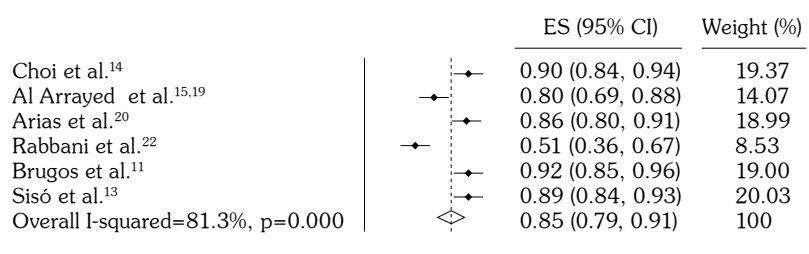

The mean age of patients with SLE at renal biopsy was reported by four studies ${ }^{11,23-25}$ to be 31.9, 27, 28.7 and 31.8 years, respectively (Tables 1 and 3). Furthermore, two other studies $^{9,10}$ reported the mean age of patients with SLE at renal involvement to be 29 and 28 years (Table 1), while a different study ${ }^{13}$ reported the mean age of patients with SLE at LN diagnosis to be 31.1 years (Table 1). Thus, all these studies reported mean values in the region of 30 years of age. In addition, two further studies ${ }^{26,27}$ described the mean age of patients at SLE diagnosis to be 26 and 33 years (Table 3).

The sex distribution in patients with BPLN was reported by 11 studies, ${ }^{11,13-15,19,20,22-27}$ with the percentage of females ranging from 51.4\% to $92.5 \%$ (Tables 1, 2, and 3). The pooled prevalence of BPLN in female based on a randomeffects model was $85 \%$ (95\% CI: 0.79 to $91 \%$; $I^{2}=81.37, \mathrm{p}<0.001$ ) (Figure 2c). A funnel plot analysis of sex distribution indicated no obvious publication bias. However, the study of Rabbani et $\mathrm{al}^{22}$ reported that the percentage of females among patients with BPLN was 51\%, significantly lower than that of the other 10 studies based on comparisons of $95 \%$ CIs (Figure 2c). The number of patients with BPLN included in the study by (b)

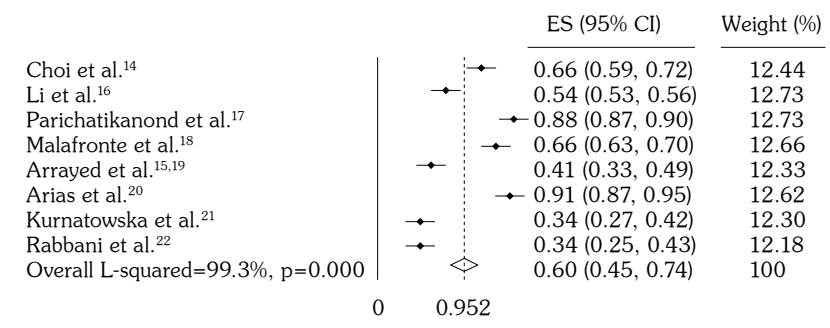

(d)

\begin{tabular}{|c|c|c|c|}
\hline & & ES $(95 \%$ CI) & Weight $(\%$ \\
\hline Neumann et al. ${ }^{9}$ & & $0.48(0.38,0.57)$ & 17.86 \\
\hline Huong et al. ${ }^{10}$ & & $0.28(0.21,0.35)$ & 21.19 \\
\hline Brugos et al. ${ }^{11}$ & $\rightarrow$ & $0.46(0.36,0.56)$ & 17.00 \\
\hline Al Arfaj et al. ${ }^{12}$ & & $0.42(0.36,0.48)$ & 22.63 \\
\hline Sisó et al. ${ }^{13}$ & $\rightarrow$ & $0.38(0.31,0.45)$ & 21.31 \\
\hline \multirow[t]{2}{*}{ Overall L-squared $=74.8 \%, p=0.003$} & . & $0.40(0.30,0.47)$ & 100 \\
\hline & 0.572 & & \\
\hline
\end{tabular}

Figure 2. Forest plots of epidemiologic data related to patients with biopsy-proven lupus nephritis. (a) The incidence of biopsy-proven lupus nephritis (with 95\% CI) in patients with systemic lupus erythematosus. (b) The incidence of biopsy-proven lupus nephritis (with 95\% CI) in patients with secondary glomerular diseases. (c) The percentage (with 95\% CI) of incidence of biopsy-proven lupus nephritis among patients with systemic lupus erythematosus and secondary glomerular diseases in female subjects. (d) Study effect estimates (with 95\% CI) and population sizes for the rate of class IV nephritis among patients with biopsy-proven lupus nephritis. ES: Estimated incidence; CI: Confidence interval. 
Rabbani et al. ${ }^{22}$ was only 37, lower than that in the other 10 studies. ${ }^{11,13-15,19,20,22-27}$ Since this may have resulted in significant heterogeneity among the 11 studies, a subsequent analysis, which had been omitted in the study of Rabbani et al., ${ }^{22}$ was performed. The results of this analysis showed that the remaining 10 studies were homogeneous $\left(I^{2}=33.1 \%, p=0.143\right)$. The pooled rate for the percentage of females in these 10 studies, based on a fixed-effects model, was $88 \%$ (95\% CI: 86-90\%), not significantly different from the overall rate calculated for all 11 studies. Five studies ${ }^{9-13}$ reported details of the distribution of the six pathologic types of BPLN (Table 1). Three studies utilized the 1982 World Health Organization criteria for the pathologic classification of LN, while two used the 2003 World Health Organization criteria. ${ }^{28}$ The distribution of the six pathologic types of BPLN were as follows: class I, 0.9-4.2\%; class II, 9.3-21.5\%; class III, $11.2-24.2 \%$; class IV, 27.847.7\%; class V, 12.1-20.3\%; and class VI, 1.3$4.7 \%$. Class IV was the most common BPLN class in each of the five studies, and the proportion of patients with class IV disease did not differ significantly between different regions, based on comparisons of $95 \%$ CIs. The pooled estimate of the percentage of class IV nephritis among patients with BPLN was 40\% (95\% CI: 33-47\%, $I^{2}=74.8 \%, \quad p<0.01$ ) (Figure 2d). Sensitivity analyses revealed that the exclusion of any study did not significantly alter the pooled estimate. Six articles ${ }^{13,23-27}$ reported data concerning the prognosis of patients with BPLN (Table 3). Five-, 10- and 20-year survival rates were reported by four, five, and three articles, respectively. The overall 5-, 10-, and 20-year survival rates were 94\% (95\% CI: $\left.88-99 \%, I^{2}=86.2 \%, p<0.001\right)$, 86\% (95\% CI: 79-92\%, $\left.I^{2}=84.8 \%, \mathrm{p}<0.001\right)$ and 71\% (95\% CI: 54-88\%, $\left.I^{2}=91.0 \%, \mathrm{p}<0.001\right)$, respectively (Figure 3). Subgroup analysis revealed that the 5-, 10-, and 20-year survival rates in the USA before 1995 were only 84\% (95\% CI: 76-92\%), 72\% (95\% CI: 63-81\%) and 52\% (95\% CI: 42-62\%), respectively. These values were significantly lower than the respective overall 5-, 10-, and 20-year survival rates in other countries after 1995: 96\% (95\% CI: 92-100\%, $I^{2}=78.2 \%$, $\mathrm{p}<0.05), 89 \% \quad\left(95 \% \mathrm{CI}: 84-94 \%, I^{2}=73.3 \%\right.$, $\mathrm{p}<0.05)$, and $80 \%$ (95\% CI: 75-85\%, $I^{2}=0 \%$, $\mathrm{p}=1$ ) (Figure 3). In addition, it was found that the survival rate of patients with BPLN in China was not lower than that in four developed countries, based on comparisons of 95\% Cls (Figure 3).

Four ${ }^{13,23,24,27}$ of the six studies reported that renal failure was the main cause of death in patients with BPLN, and that the following parameters were risk factors for renal failure in patients with BPLN: class IV nephritis; male sex; hypertension; nephrotic syndrome; thrombocytopenia; hypocomplementemia; abnormal urinalysis; elevated anti-DNA antibody titers; and decreased creatinine clearance rate (Table 3).

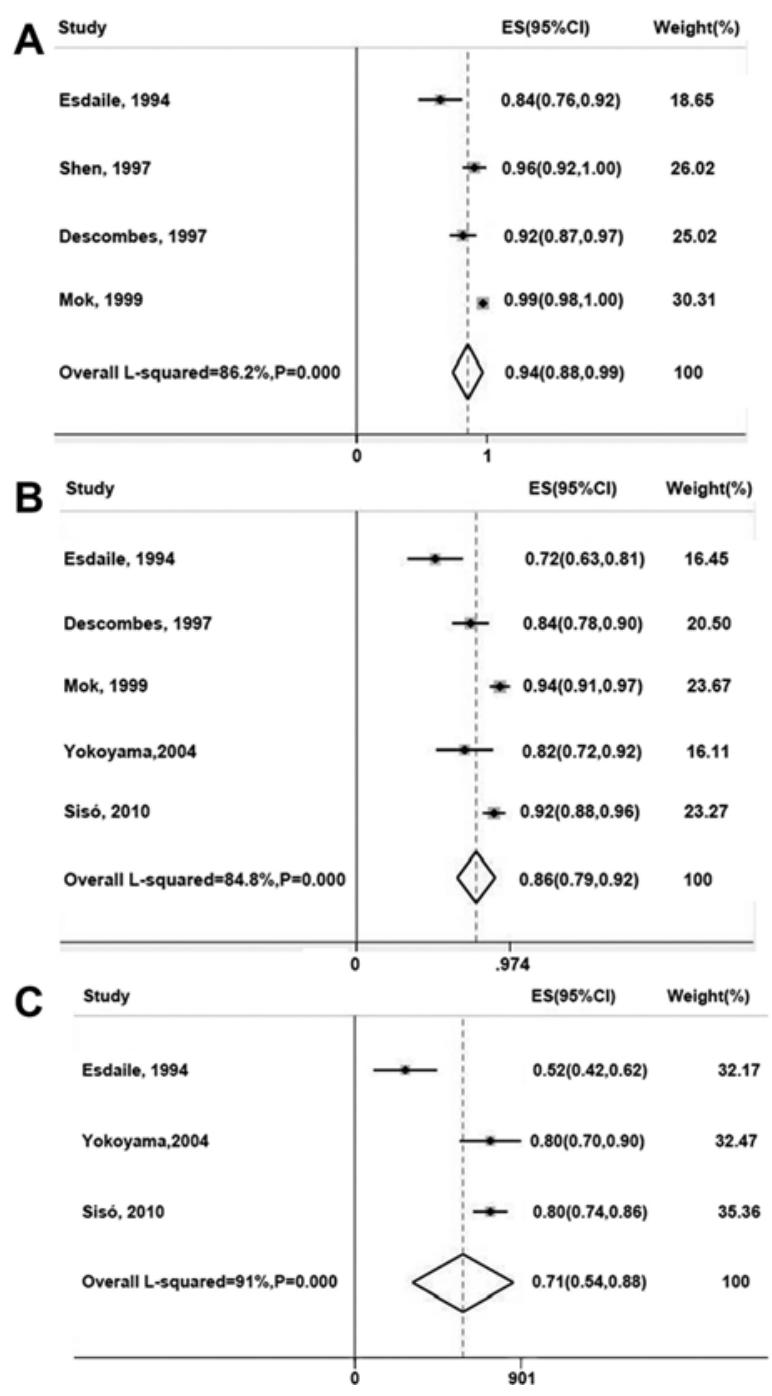

Figure 3. Forest plots of study effect estimates and population sizes for survival rates of patients with biopsyproven lupus nephritis at five (a), 10 (b) and 20 (c) years. ES: Estimated percentage; CI: Confidence interval. 


\section{DISCUSSION}

The present meta-analysis was designed with the aim of improving our understanding of the epidemiology of BPLN. Although clinical findings such as proteinuria, hematuria, and elevated serum creatinine are often used for the diagnosis of $\mathrm{LN}^{29}$ these clinical presentations are not suitable for establishing the true incidence of LN. ${ }^{5}$ Renal pathology is recognized as the gold standard for the diagnosis of $\mathrm{LN}$, but to date, only limited epidemiologic data related to BPLN have been published. By conducting a metaanalysis of relevant articles concerning BPLN, the present study has provided novel insights into the epidemiologic characteristics of BPLN. BPLN developed in about 29\% of patients with SLE and accounted for almost $60 \%$ of SGD in renal biopsy databases. The mean age of patients with SLE was approximately 30 years at renal biopsy, and $85 \%$ of patients with BPLN were female. The overall 5-, 10-, and 20-year survival rates for patients with BPLN were 94\%, 86\% and $71 \%$, respectively. Class IV nephritis, present in $40 \%$ of patients with BPLN, was a risk factor for renal failure that contributed to poor prognosis in patients with SLE.

The prevalence rates of renal involvement in patients with SLE show regional variation, 4,30 and the renal biopsy rates in these patients also differ between hospitals. In order to objectively demonstrate the epidemiologic prevalence rate of BPLN among patients with SLE and minimize the influence of differing renal biopsy rates, we specifically incorporated cohort studies in which renal biopsies were performed routinely on most patients with LN. In our study, we found that almost one-third of patients with SLE had BPLN, with the prevalence rate of BPLN higher in Asia than in Europe and the USA. Moreover, we also found that BPLN was the most common SGD in each available renal biopsy database, emphasizing the importance of BPLN in the etiology of SGD. The great heterogeneity in the incidence of BPLN among patients with SLE or SGD may be related not only to the different prevalence rates of $\mathrm{LN}$ in distinct regions, but also to the varied profiles of SGD in different countries. For example, diabetic nephropathy was less common in developing countries than in developed countries. ${ }^{18,21}$
The sex distribution and ages of patients with BPLN may also vary with ethnicity. In Pakistan, the percentage of female patients with BPLN was $51.4 \%,{ }^{22}$ compared with $84.6 \%$ in Northwest England. ${ }^{30}$ Among Afro-Caribbean and Chinese females, the prevalence rate of $\mathrm{LN}$ was highest in those aged 20-39 years, whereas among white and Indo-Asian females, it was highest in those aged 40-59 years. ${ }^{30}$ Our analysis revealed that females, especially those aged 20-30 years, represented the majority of patients with BPLN, indicating that renal involvement of SLE was a common complication in female patients of childbearing age.

Class IV nephritis was identified as the most frequent pathologic type in all studies that examined this (Table 1), although class II, class III and class $\mathrm{V}$ were also common; in contrast, class I and class VI were more rarely seen. A possible explanation for this is that patients with SLE and LN class I have milder presentations that are not indicators for renal biopsy, such that renal biopsy is less commonly undertaken. In contrast, patients with class II, class III, class IV or class V LN would likely have more obvious presentations, and hence would be more likely to undergo renal biopsy. Most patients with LN class VI would have end-stage renal failure, making renal biopsy unnecessary. However, it is not known why class IV was seen more frequently than class II, class III and class V; it is possible that this is related to an undiscovered pathologic mechanism.

Improvements in diagnostics and supportive treatments may have prolonged the life expectancy of patients with LN. ${ }^{7,8}$ The present study indicated that the prognosis of patients with BPLN had improved greatly during the past 20 years, although long-term survival remains unsatisfactory. Recently, a meta-analysis of observational studies from the $1950 \mathrm{~s}$ to the $2000 \mathrm{~s}^{31}$ demonstrated that the overall five-year survival rate of patients with SLE had increased from 74.8 to $94.8 \%$, and the 10-year survival rate had increased from $63.2 \%$ to $91.4 \%$. Furthermore, the prevalence of renal damage, an important cause of unsatisfactory prognosis in patients with SLE, had not changed significantly over the past 50 years, unlike neuropsychiatric damage. Renal failure was shown by our study to be the most common cause of death in patients with BPLN, and hence contributed to the mortality associated with SLE. 
Cardiovascular events are also an important cause of mortality in patients with BPLN. Interestingly, two recent studies reported that early-onset LN and end-stage LN were significantly related to cardiovascular events in patients with SLE. ${ }^{32,33}$ Sinicato et al. ${ }^{34}$ reported that LN could accelerate atherosclerosis in patients with SLE, leading to the development of cardiovascular events. Our study also identified infection, partially attributable to the use of corticosteroids and cytotoxic agents for the remission of $\mathrm{LN}$, as an important cause of mortality in patients with BPLN.

The present study also explored the risk factors for renal failure in patients with BPLN. Class IV glomerulonephritis was found to be the most frequent risk factor. A recent study ${ }^{35}$ indicated that class IV glomerulonephritis was the most ominous pathologic type among all the classes of nephritides, that $15-30 \%$ of patients with class IV nephritis did not reach remission, and that $15-30 \%$ of those reaching remission suffered relapse. Moreover, male sex, hypertension, nephrotic syndrome, hypocomplementemia, abnormal urinalysis, elevated anti-DNA antibody titers, and decreased creatinine clearance rate were all shown to be risk factors for renal failure. However, although these clinical parameters can warn of the possibility of renal involvement in patients with SLE, they are not sufficiently sensitive or specific to detect ongoing disease activity in lupus kidneys or early relapse of LN. Therefore, renal biopsy or other novel biomarkers are necessary to enhance the diagnostic accuracy for LN, detect early renal exacerbations, monitor treatment response, and evaluate prognosis.

Incidence studies of BPLN from the all the population was not available. Significant heterogeneity observed in the most of the analysis explains the differences in the study conduct, study design, methodology and bias in the studies included in the meta-analysis. We could not conduct the meta-regression analysis to test the differences in the incidence of BPLN in different ethnic groups due to insufficient number of studies available for the meta-regression analysis.

In conclusion, to the best of our knowledge, this is the first meta-analysis to systematically review the epidemiologic characteristics of BPLN. Our findings demonstrate that $\mathrm{LN}$ is a common complication of young female patients with SLE, and is the most prevalent etiology of SGD in renal biopsy databases. In addition, the prevalence rates of BPLN among patients with SLE or SGD show regional variation. Furthermore, the prognosis of patients with BPLN is still unsatisfactory in spite of the great improvement during the past 20 years. It is concluded that more attention should be paid to class IV nephritis due to its high frequency and association with a poor prognosis.

\section{Declaration of conflicting interests}

The authors declared no conflicts of interest with respect to the authorship and/or publication of this article.

\section{Funding}

This work was supported by Lin He's New Medicine and Clinical Translation Academician Workstation Research Fund (Grant No: 17331208), Wenzhou Science and technology bureau (Grant No: H20150006), Zhejiang province traditional Chinese medicine technology plan (Grant No: 2015ZB077).

\section{REFERENCES}

1. Kamen DL. Environmental influences on systemic lupus erythematosus expression. Rheum Dis Clin North Am 2014;40:401-12.

2. Fortuna G, Brennan MT. Systemic lupuserythematosus: epidemiology, pathophysiology, manifestations, and management. Dent Clin North Am 2013;57:631-55.

3. Gurevitz SL, Snyder JA, Wessel EK, Frey J, Williamson BA. Systemic lupus erythematosus: a review of the disease and treatment options. Consult Pharm 2013;28:110-21.

4. Zubair A, Frieri M. Lupus nephritis: review of the literature. Curr Allergy Asthma Rep 2013;13:580-6.

5. Zabaleta-Lanz M, Vargas-Arenas RE, Tápanes F, Daboin I, Atahualpa Pinto J, Bianco NE. Silent nephritis in systemic lupus erythematosus. Lupus 2003;12:26-30.

6. Lech M, Anders HJ. The pathogenesis of lupus nephritis. J Am Soc Nephrol 2013;24:1357-66.

7. Giannico G, Fogo AB. Lupus nephritis: is the kidney biopsy currently necessary in the management of lupus nephritis? Clin J Am Soc Nephrol 2013;8:138-45.

8. Houssiau FA, Lauwerys BR. Current management of lupus nephritis. Best Pract Res Clin Rheumatol 2013;27:319-28.

9. Neumann K, Wallace DJ, Azen C, Nessim S, Fichman M, Metzger AL, et al. Lupus in the 1980s: III. Influence of clinical variables, biopsy, and treatment on the outcome in 150 patients with lupus nephritis seen at a single center. Semin Arthritis Rheum 1995;25:47-55. 
10. Huong DL, Papo T, Beaufils H, Wechsler B, Blétry $\mathrm{O}$, Baumelou $\mathrm{A}$, et al. Renal involvement in systemic lupus erythematosus. A study of 180 patients from a single center. Medicine (Baltimore) 1999;78:148-66.

11. Brugos B, Kiss E, Szodoray P, Szegedi G, Zeher M. Retrospective analysis of patients with lupus nephritis: data from a large clinical immunological center in Hungary. Scand J Immunol 2006;64:433-7.

12. Al Arfaj AS, Khalil N, Al Saleh S. Lupus nephritis among 624 cases of systemic lupus erythematosus in Riyadh, Saudi Arabia. Rheumatol Int 2009;29:105767.

13. Sisó A, Ramos-Casals M, Bové A, Brito-Zerón P, Soria N, Nardi N, et al. Outcomes in biopsy-proven lupus nephritis: evaluation of 190 white patients from a single center. Medicine (Baltimore) 2010;89:300-7.

14. Choi IJ, Jeong HJ, Han DS, Lee JS, Choi KH, Kang SW, et al. An analysis of 4,514 cases of renal biopsy in Korea. Yonsei Med J 2001;42:247-54.

15. Al Arrayed A, George SM, Malik AK, Al Arrayed S, Rajagopalan S, Al Arrayed A, et al. The spectrum of glomerular diseases in the kingdom of bahrain: an epidemiological study based on renal biopsy interpretation. Transplant Proc 2004;36:1792-5.

16. Li LS, Liu ZH. Epidemiologic data of renal diseases from a single unit in China: analysis based on 13,519 renal biopsies. Kidney Int 2004;66:920-3.

17. Parichatikanond P, Chawanasuntorapoj R, Shayakul C, Choensuchon B, Vasuvattakul S, Vareesangthip $\mathrm{K}$, et al. An analysis of 3,555 cases of renal biopsy in Thailand. J Med Assoc Thai 2006;89 Suppl 2:S106-11.

18. Malafronte P, Mastroianni-Kirsztajn G, Betônico GN, Romão JE Jr, Alves MA, Carvalho MF, et al. Paulista Registry of glomerulonephritis: 5-year data report. Nephrol Dial Transplant 2006;21:3098-105.

19. Arrayed AA, Shariff S, Maamari MM. Kidney disease in Bahrain: a biopsy-based epidemiological study. Transplant Proc 2007;39:875-8.

20. Arias LF, Henao J, Giraldo RD, Carvajal N, Rodelo J, Arbeláez M. Glomerular diseases in a Hispanic population: review of a regional renal biopsy database. Sao Paulo Med J 2009;127:140-4.

21. Kurnatowska I, Jedrzejka D, Malyska A, WagrowskaDanilewicz M, Danilewicz M, Nowicki M. Trends in the incidence of biopsy-proven glomerular diseases in the adult population in central Poland in the years 1990-2010. Kidney Blood Press Res 2012;35:254-8.

22. Rabbani MA, Memon GM, Ahmad B, Memon S, Tahir SA, Tahir S. Percutaneous renal biopsy results: a retrospective analysis of 511 consecutive cases. Saudi
J Kidney Dis Transpl 2012;23:614-8.

23. Esdaile JM, Abrahamowicz M, MacKenzie T, Hayslett JP, Kashgarian M. The time-dependence of longterm prediction in lupus nephritis. Arthritis Rheum 1994;37:359-68.

24. Shen K, Yu Y, Tang Z, Liu Z, Li L. The prognosis of biopsy-proven lupus nephritis in chinese patients: long term follow-up of 86 cases. Chin Med J (Engl) 1997;110:502-7.

25. Descombes E, Droz D, Drouet L, Grünfeld JP, Lesavre P. Renal vascular lesions in lupus nephritis. Medicine (Baltimore) 1997;76:355-68.

26. Mok CC, Wong RW, Lau CS. Lupus nephritis in Southern Chinese patients: clinicopathologic findings and long-term outcome. Am J Kidney Dis 1999;34:315-23.

27. Yokoyama H, Wada T, Hara A, Yamahana J, Nakaya $\mathrm{I}$, Kobayashi $\mathrm{M}$, et al. The outcome and a new ISN/RPS 2003 classification of lupus nephritis in Japanese. Kidney Int 2004;66:2382-8.

28. Weening JJ, D'Agati VD, Schwartz MM, Seshan SV, Alpers CE, Appel GB, et al. The classification of glomerulonephritis in systemic lupus erythematosus revisited. J Am Soc Nephrol 2004;15:241-50.

29. Saxena R, Mahajan T, Mohan C. Lupus nephritis: current update. Arthritis Res Ther 2011;13:240.

30. Patel M, Clarke AM, Bruce IN, Symmons DP. The prevalence and incidence of biopsy-proven lupus nephritis in the UK: Evidence of an ethnic gradient. Arthritis Rheum 2006;54:2963-9.

31. Mak A, Cheung MW, Chiew HJ, Liu Y, Ho RC. Global trend of survival and damage of systemic lupus erythematosus: meta-analysis and meta-regression of observational studies from the 1950s to 2000s. Semin Arthritis Rheum 2012;41:830-9.

32. Faurschou M, Mellemkjaer L, Starklint H, Kamper AL, Tarp U, Voss A, et al. High risk of ischemic heart disease in patients with lupus nephritis. J Rheumatol 2011;38:2400-5.

33. Ward MM. Cardiovascular and cerebrovascular morbidity and mortality among women with endstage renal disease attributable to lupus nephritis. Am J Kidney Dis 2000;36:516-25.

34. Sinicato NA, da Silva Cardoso PA, Appenzeller S. Risk factors in cardiovascular disease in systemic lupus erythematosus. Curr Cardiol Rev 2013;9:15-9.

35. Ferraccioli G, Gremese E. Class IV-G and IV-S lupus nephritis, interstitial infiltrates and prognosis: state of the art and unmet medical needs. G Ital Nefrol 2012;29:655-60. [Abstract] 